\title{
Climat et culture
}

\section{Carlos B. Quinto}

Dr méd., membre du Comité central de la FMH, responsable du département Santé publique et professions de la santé

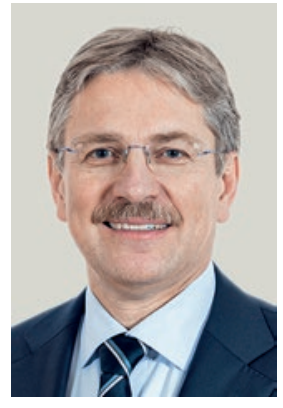

Les tendances à long terme avec l'augmentation des journées tropicales, des fortes pluies, de certaines maladies et des vecteurs les transmettant ne sont pas la conséquence de fluctuations météorologiques mais de changements climatiques. Ces dernières années, les conséquences sur nos patientes et patients souffrant par exemple de maladies cardiaques et rénales préexistantes se sont fait nettement ressentir dans nos consultations: adaptation préventive du comportement, adaptation à court terme de la médication lors des journées tropicales. Le lien entre santé et climat étant évident et établi scientifiquement, on comprend aisément pourquoi la Chambre médicale du 7 octobre 2021 a approuvé le document de base «Santé planétaire: stratégie du corps médical suisse sur les possibilités d'action concernant le changement climatique».

\section{L'unité sera décisive dans les années à venir pour la promotion de la santé publique en Suisse.}

La stratégie a été développée sur une large base au sein de la FMH, avec le soutien scientifique de l'Institut tropical et de santé publique suisse; sa mise en œuvre a été confiée au département Santé publique, professions de la santé et produits thérapeutiques. Nous espérons que toutes les parties concernées poursuivront leur participation constructive et engagée et en profitons pour remercier tous les membres du groupe de travail et les organisations qu'ils représentent. Vous trouverez un article détaillé dans ce numéro du BMS, à la page 1394. Cette décision prise lors de la dernière Chambre médicale a également permis de signaler que le corps médical est uni et mène une action intergénérationnelle.

C'est précisément cette unité qui sera décisive dans les années à venir. La pandémie a montré que la Suisse est en bien moins bonne posture dans le domaine de la santé publique que dans celui des soins, la structure fédérale constituant certainement un défi en cas de pandémie. L'essentiel de la responsabilité incombe cependant au conseiller fédéral compétent, à ses décisions en matière de personnel, et également au climat, ici au sens d'une culture, qui n'est favorable ni à la santé pu- blique, ni au maintien d'un accès à des soins de bonne qualité y compris en médecine de famille. Un examen critique de l'approche de l'administration est en cours. Quel développement la pandémie a-t-elle connu en Suisse depuis début 2020? Comment l'incidence a-telle évolué et évolue-t-elle aujourd'hui? et la mortalité? L'Office fédéral de la statistique pourrait fournir des données instructives à ce sujet, en incluant une comparaison avec d'autres pays européens. A quel degré les hôpitaux et les unités de soins intensifs ont-ils été occupés? Si les rapports hebdomadaires sur le site de l'Office fédéral de la santé publique fournissent des informations, il serait intéressant d'avoir une vue d'ensemble chronologique des mesures prises mais aussi de celles qui n'ont pas été prises.

Des différences très importantes ont régné entre les cantons au niveau de la gestion de la crise, de l'inclusion du corps médical, du dépistage et de la vaccination. Il existe ainsi aujourd'hui des cabinets médicaux qui n'ont encore jamais eu la possibilité de vacciner ou d'autres qui ont cessé de vacciner en raison d'obstacles administratifs et de réglementations éloignées de la pratique. Un potentiel d'amélioration existe au niveau de la collaboration avec les professions médicales et paramédicales afin de mieux pouvoir faire face aux défis de cette pandémie. Du point de vue de la santé publique, une participation et une coopération adéquates en sont une condition préalable. La Loi sur les épidémies est également meilleure que sa réputation le laisse entendre, si elle est correctement appliquée. En Suisse, le système de santé est en grande partie géré

\section{Les cantons incluant activement leur société} cantonale de médecine sont plus avancés dans la lutte contre la pandémie.

par les cantons, et il s'est avéré que les cantons qui ont activement inclus leur société cantonale de médecine sont plus avancés dans leur lutte contre la pandémie: alors que certains cantons rattrapent les erreurs commises au niveau national, d'autres les accentuent. Rester passif et espérer un «changement climatique» ne suffit malheureusement plus. Une unité et une action proactive de la part du corps médical sont nécessaires. 